\title{
Cardiovascular magnetic resonance imaging findings in Ebstein anomaly
}

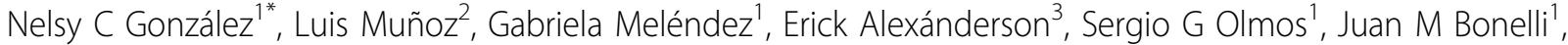 \\ Aloha Meave ${ }^{1}$, Luis Marroquin ${ }^{3}$, Fernando Iñarra ${ }^{3}$, José E Telich-Tarriba ${ }^{3}$ \\ From 15th Annual SCMR Scientific Sessions \\ Orlando, FL, USA. 2-5 February 2012
}

\section{Background}

In recent years, cardiac magnetic resonance (CMR) imaging has emerged as the reference standard for cardiac imaging in patients with many forms of congenital heart disease, in particular for lesions that affect the right ventricle. Few studies have been published in assessment of Ebstein anomaly by CMR imaging.

\section{Methods}

CMR studies of patients with EA conducted from June 2007 to June 2011 were analized. Cine spin echo images in multiple views, and angiography were evaluated to assess chambers morphology and diameters, wall thickness and left ventricular function, also were measured the atrialized right ventricular portion and the functional tricuspid valve position. And Inversion Recovery sequence for determination of fibrosis.

\section{Results}

Ninety four patients were studied. Table 1 summarizes the main results. Tricuspid valve regurgitation was found in $96.9 \%$, severe in $62.5 \%$ of the cases. The presence of left ventricle fibrosis was significant associated with right ventricle longest dimension including the atrialized portion $(\mathrm{p}=0.003)$, the diastolic and systolic diameters $(\mathrm{p}=0.011$ and $\mathrm{p}=0.005)$.

\section{Conclusions}

CMR findings in EA were demonstrated. There was a clear positive relationship between the presence of LV fibrosis and the right ventricle dimensions.

${ }^{1}$ Cardiovascular Magnetic Resonance Imaging, National Institute of Cardiology "Ignacio Chávez", Mexico City, Mexico

Full list of author information is available at the end of the article

\section{Funding}

National Institute of Cardiology "Ignacio Chávez", Mexico City, Mexico.

Table 1 Results

\begin{tabular}{|c|c|c|}
\hline Variable & Mean & SD \\
\hline Age (years) & 23.0 & 13.02231 \\
\hline $\mathrm{LVdV}(\mathrm{mm})$ & 36.5 & 8.36997 \\
\hline LVsD (mm) & 25.7 & 6.55335 \\
\hline $\mathrm{dS}(\mathrm{mm})$ & 7.9 & 10.5891 \\
\hline Lateral wall diastolic (mm) & 6.2 & 1.42729 \\
\hline $\operatorname{RVdD}(\mathrm{mm})$ & 55.6 & 20.61004 \\
\hline RVsD (mm) & 47.6 & 21.16745 \\
\hline Right ventricular longitudinal diameter (mm) & 96.1 & 22.96363 \\
\hline RVfw $(\mathrm{mm})$ & 4.5 & 1.40918 \\
\hline RA U/L (mm) & 66.0 & 22.36908 \\
\hline RA $M / L(m m)$ & 63.9 & 20.99671 \\
\hline LA U/L (mm) & 44.1 & 16.28296 \\
\hline LA M/L & 36.6 & 14.03464 \\
\hline LVEF (\%) & 47.2 & 10.96851 \\
\hline LV mass (gr) & 60.0 & 28.60894 \\
\hline LVEDV (ml) & 78.1 & 34.64511 \\
\hline $\operatorname{LVESV}(\mathrm{ml})$ & 40.7 & 18.73527 \\
\hline Left ventricular stroke volume (ml) & 36.6 & 19.18734 \\
\hline Atrialized portion of right ventricle (mm) & 56.1 & 26.3945 \\
\hline Atrialized portion of right ventricle (\%) & 59.3 & 19.84599 \\
\hline Atrial septal defect $(\mathrm{mm})$ & 5.7 & 6.98696 \\
\hline Pulmonary artery (mm) & 19.6 & 6.87248 \\
\hline Right pulmonary branch (mm) & 12.8 & 4.50256 \\
\hline Left pulmonary branch (mm) & 13.3 & 4.49312 \\
\hline
\end{tabular}

LVdD: Left ventricular diastolic diameter; LVsD: Left ventricular systolic diameter; dS: diastolic septum; RVdD: right ventricular diastolic diameter; $\mathrm{RVsD}$ : right ventricular systolic diameter; RVfw: right ventricular free Wall; RA $\mathrm{U} / \mathrm{L}$ : right atrium upper/lower; RA M/L: right atrium mid/lateral; LA U/L: left atrium upper/lower; LA M/L: mid/lateral; LVEF: ejection fraction; LV: left ventricular; LVEDVI: end-diastolic volume; LVESVI: end-systolic volume.

\section{() Biomed Central}




\section{Author details}

${ }^{1}$ Cardiovascular Magnetic Resonance Imaging, National Institute of

Cardiology "Ignacio Chávez", Mexico City, Mexico. ${ }^{2}$ Embryology, National Institute of Cardiology "Ignacio Chávez", Mexico City, Mexico. ${ }^{3}$ Nuclear Cardiology, National Institute of Cardiology "Ignacio Chávez", Mexico City, Mexico.

Published: 1 February 2012

doi:10.1186/1532-429X-14-S1-P107

Cite this article as: González et al.: Cardiovascular magnetic resonance

imaging findings in Ebstein anomaly. Journal of Cardiovascular Magnetic Resonance 2012 14(Suppl 1):P107.

Submit your next manuscript to BioMed Central and take full advantage of:

- Convenient online submission

- Thorough peer review

- No space constraints or color figure charges

- Immediate publication on acceptance

- Inclusion in PubMed, CAS, Scopus and Google Scholar

- Research which is freely available for redistribution

Submit your manuscript at www.biomedcentral.com/submit
() Biomed Central 